\title{
Observation of Cold, Long-Lived Antiprotonic Helium Ions
}

\author{
M. Hori, ${ }^{1}$ J. Eades, ${ }^{2}$ R. S. Hayano, ${ }^{2}$ W. Pirkl, ${ }^{2}$ E. Widmann, ${ }^{2}$ H. Yamaguchi, ${ }^{2}$ H. A. Torii, ${ }^{3}$ B. Juhász, ${ }^{4}$ D. Horváth, ${ }^{5}$ \\ K. Suzuki, ${ }^{6}$ and T. Yamazaki ${ }^{7}$ \\ ${ }^{1}$ CERN, CH-1211 Geneva 23, Switzerland \\ ${ }^{2}$ Department of Physics, University of Tokyo, 7-3-1 Hongo, Bunkyo-ku, Tokyo 113-0033, Japan \\ ${ }^{3}$ Institute of Physics, University of Tokyo, Komaba, Meguro-ku, Tokyo 153-8902, Japan \\ ${ }^{4}$ Institute of Nuclear Research of the Hungarian Academy of Sciences, H-4001 Debrecen, Hungary \\ ${ }^{5}$ KFKI Research Institute for Particle and Nuclear Physics, H-1525 Budapest, Hungary \\ ${ }^{6}$ Physik-Department, Technische Universität München, D-85747 Garching, Germany \\ ${ }^{7}$ Heavy Ion Nuclear Physics Laboratory, RIKEN, Wako, Saitama 351-0198, Japan
}

(Received 31 July 2004; published 14 February 2005)

Cold, two-body antiprotonic helium ions $\bar{p}^{4} \mathrm{He}^{2+}$ and $\bar{p}^{3} \mathrm{He}^{2+}$ with 100-ns-scale lifetimes, occupying circular states with the quantum numbers $n_{i}=28-32$ and $\ell_{i}=n_{i}-1$ have been observed. They were produced by cooling three-body antiprotonic helium atoms in an ultra-low-density helium target at temperature $T \sim 10 \mathrm{~K}$ by atomic collisions, and then removing their electrons by inducing a laser transition to an autoionizing state. The lifetimes of $\bar{p}^{3} \mathrm{He}^{2+}$ against annihilation induced by collisions were shorter than those of $\bar{p}^{4} \mathrm{He}^{2+}$, and decreased for larger- $n_{i}$ states.

DOI: 10.1103/PhysRevLett.94.063401

PACS numbers: $36.10 .-\mathrm{k}, 25.43 .+\mathrm{t}, 34.90 .+\mathrm{q}$

The antiprotonic helium ion $\left(\bar{p} \mathrm{He}^{2+}\right)$ [1,2] is a singly charged, two-body Coulomb system composed of an antiproton and a helium nucleus. We here report on the first production of cold ions of the $\bar{p}^{4} \mathrm{He}^{2+}$ and $\bar{p}^{3} \mathrm{He}^{2+}$ isotopes with lifetimes $\left(\tau_{i} \sim 100 \mathrm{~ns}\right)$ against annihilation. These highly excited circular states, which had principal and angular momentum quantum numbers $n_{i}=28-32$ and $\ell_{i}=n_{i}-1$, were selectively populated by cooling threebody antiprotonic helium atoms by atomic collisions, and then removing their electrons by inducing a laser transition to an autoionizing state.

These ions constitute ideal semiclassical Bohr systems whose spin-independent parts of the energy levels (left side of Fig. 1) can be theoretically calculated to very high precision $\left(\sim 10^{-8}\right)$ using the simple equation [3]

$$
E_{n}=-\frac{4 R_{\infty} h c}{n_{i}^{2}} \frac{M}{m_{e}} \frac{Q_{\bar{p}}^{2}}{e^{2}}
$$

where the reduced mass of the system is denoted by $M$, the electron mass by $m_{e}$, the Rydberg constant by $R_{\infty}$, and the antiproton and electron charges by $Q_{\bar{p}}$ and $e$. For comparison, in the $2 p$ state of atomic hydrogen relativistic corrections to the above equation appear at a level of $\sim 10^{-5}$ and QED corrections at $\sim 10^{-6}[3]$. These effects are very small in the $\bar{p} \mathrm{He}^{2+}$ case, partly because of the large mass and small magnetic moment of the antiproton, and partly because the probability density of its semiclassical orbits are circular and have almost no overlap with the helium nucleus. A single isolated ion can easily be shown to have typical lifetimes near $n_{i}=30$ of $\tau_{i} \sim 0.3-0.4 \mu$ s against annihilation, as the antiproton undergoes a series of radiative transitions [3] of the type $\Delta n_{i}=\Delta \ell_{i}=-1$ (indicated
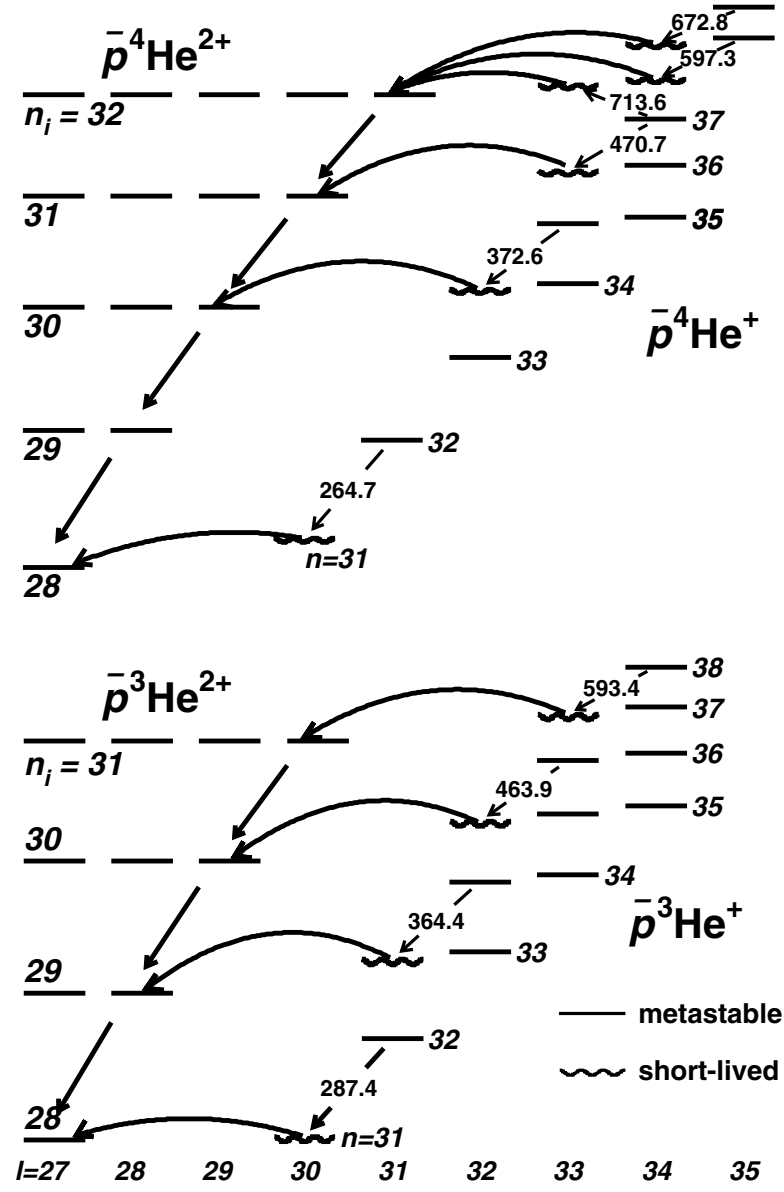

FIG. 1. Portion of energy level diagram of $\bar{p} \mathrm{He}^{2+}$ ions and $\bar{p} \mathrm{He}^{+}$atoms. Wavelengths of the laser transitions are indicated in nanometers The curved arrows indicate Auger transitions to ionic states, and the straight arrows radiative $\bar{p} \mathrm{He}^{2+}$ transitions. 
with straight arrows in Fig. 1), before being absorbed by the nucleus from the $n_{i} \sim 3$ region [4].

The antiproton mass and charge were recently determined to a precision of one part in $10^{8}$ by combining the results of laser spectroscopy experiments [5] on the neutral three-body $\bar{p} \mathrm{He}^{+} \equiv \bar{p}+\mathrm{He}^{2+}+e^{-}$atom [6], and the cyclotron frequency of antiprotons measured in a Penning trap [7]. These derivations, however, relied heavily on the results of difficult three-body QED calculations of the $\bar{p} \mathrm{He}^{+}$atomic energy levels (right side of Fig. 1) which currently have errors $\left(\sim 10^{-8}\right)$ similar in magnitude to those of the measured laser transition frequencies $[8,9]$. This is not the case for the two-body $\bar{p} \mathrm{He}^{2+}$, where the long lifetime necessary for laser spectroscopy is coupled with extreme ease in calculating its energy levels; these factors may make this ion a better candidate than the $\bar{p} \mathrm{He}^{+}$ atom for determining the properties of antiprotons at the highest precision.

Antiprotons coming to rest in helium can produce $\bar{p} \mathrm{He}^{2+}$ ions in doubly ionizing collisions with helium atoms when their kinetic energy falls to $E \sim 10 \mathrm{eV}$ $[10,11]$. The produced ions recoil with kinetic energy corresponding to temperature $T \sim 10^{4} \mathrm{~K}[10,11]$ and must come to thermal equilibrium via numerous collisions with the surrounding gas (e.g., at $\sim 10 \mathrm{~K}$ ) if they are to be studied by high precision laser spectroscopy. Only a few such binary encounters, however, suffice for collisional Stark effects to mix the high- $\ell_{i}$ states with the $S, P$, and $D$ states [10-13], and since these have a large overlap with the helium nucleus, the ion will be normally destroyed within picoseconds $[4,12-15]$.

A two-step method that solves these problems and produces cold, long-lived $\bar{p} \mathrm{He}^{2+}$ ions occupying a given state $\left(n_{i}, \ell_{i}\right)$ was suggested by the laser spectroscopy experiments with high- $(n, \ell)$ neutral $\bar{p} \mathrm{He}^{+}$atoms referred to above. These atoms reach thermal equilibrium intact and survive for several microseconds afterward because the electron inhibits collisional Stark mixing [6]. This allows transitions to Auger-dominated states $\left(n_{A}, \ell_{A}\right)$ to be subsequently induced by a laser, and a spontaneous Auger decay will then produce the nearest ionic state $\left(n_{i}, \ell_{i}\right)$ :

$$
\bar{p} \mathrm{He}_{(n, \ell)}^{+} \stackrel{h \nu}{\rightarrow} \bar{p} \mathrm{He}_{\left(n_{A}, \ell_{A}\right)}^{+} \rightarrow \bar{p} \mathrm{He}_{\left(n_{i}, \ell_{i}\right)}^{2+}+e^{-}
$$

In these $\bar{p} \mathrm{He}^{+}$experiments, the Auger decay lifetime $\tau_{\left(n_{A}, \ell_{A}\right)}^{A}$ was visible as the tail of an annihilation rate "spike" (Fig. 2) that appeared when the laser induced a transition to state $\left(n_{A}, \ell_{A}\right)$. Normally, collisional Stark mixing once again immediately destroys any ion produced subsequently by the Auger process, in which case the length of the tail measures $\tau_{\left(n_{A}, \ell_{A}\right)}^{A}$ directly (folded with the timing resolution of the laser pulse). In fact the Auger lifetime of most neutral $\bar{p} \mathrm{He}^{+}$states measured in this way does agree with theoretical values within the experimental errors $[8,9,16]$. We expected, however, that as the target

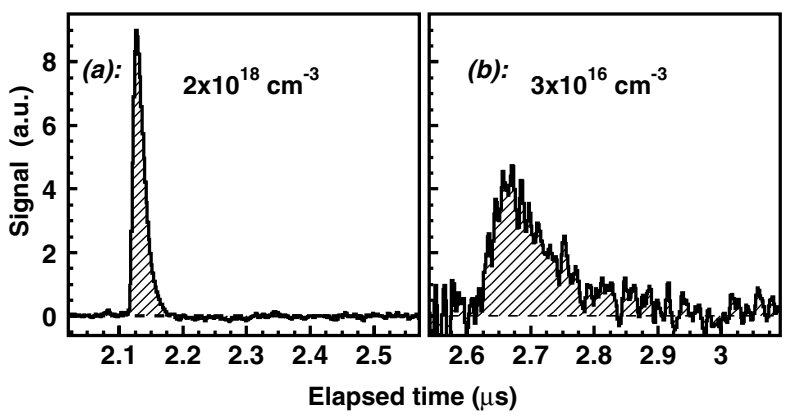

FIG. 2. Annihilation spike produced by inducing the $\bar{p}^{4} \mathrm{He}^{+}$ transition $(n, \ell)=(39,35) \rightarrow(38,34)$, measured at a high target density (a). A prolongation of the tail is observed at ultralow densities (b), indicating the formation of long-lived $\bar{p}^{4} \mathrm{He}^{2+}$ ions populating state $\left(n_{i}, \ell_{i}\right)=(32,31)$.

density (i.e., collision rate) was reduced, the $\bar{p} \mathrm{He}^{2+}$ ion's own lifetime would begin to compete with the Auger lifetime of its parent $\bar{p} \mathrm{He}^{+}$state. This would be seen as a lengthening of the tail and should therefore be an unambiguous signature of the production of cold, long-lived ions. If we assume ions thermalized to temperature $T$ to be destroyed in binary collisions with helium atoms at target density $\rho$, their decay rate will be

$$
\gamma_{i}(\rho) \sim \gamma_{i}(\rho=0)+\sigma \rho \sqrt{\frac{8 k_{B} T}{\pi M_{\mathrm{red}}}},
$$

where the cross section of collision-induced annihilation is denoted by $\sigma$, the Boltzmann constant by $k_{B}$, and the reduced mass of the $\bar{p} \mathrm{He}^{2+}-\mathrm{He}$ system by $M_{\text {red }}$. Previous experiments had revealed no such effect, but assuming a cross section of $\sigma \sim 10^{-15} \mathrm{~cm}^{-3}$, it seemed likely that the collision rate at even the lowest helium densities used heretofore $\left[\rho \sim(0.5-1.5) \times 10^{18} \mathrm{~cm}^{-3}\right.$ in Ref. [5]—itself $10^{3}-10^{4}$ times lower than in previous experiments [6] ] had been 1-2 orders of magnitude too high to produce it.

The experimental setup was similar to the one used previously to study neutral $\bar{p} \mathrm{He}^{+}$atoms [5], but the much lower target densities and higher time resolutions needed to unambiguously demonstrate the production of $\bar{p} \mathrm{He}^{2+}$ ions necessitated numerous modifications in the particle decelerator, target, detectors, and laser systems. Beam pulses containing $3 \times 10^{7}$ antiprotons with energy $E=5.3 \mathrm{MeV}$, pulse length $\Delta t=100 \mathrm{~ns}$, and repetition rate $f=0.01 \mathrm{~Hz}$ were produced by the antiproton decelerator (AD) of CERN. A radio frequency quadrupole decelerator (RFQD) [17] decelerated some $\sim 30 \%$ of the antiprotons in each pulse to energies $T \sim 80 \mathrm{keV}$; these were diverted by an achromatic momentum analyzer consisting of dipole, quadrupole, and solenoidal magnets connected to the output of the RFQD, and entered a helium gas target through a $1.3-\mu \mathrm{m}$-thick polyethylene naphthalate window. The target was a 15 -cm-diameter, 30-cm-long cylindrical chamber filled with ${ }^{4} \mathrm{He}$ (purity $99.9999 \%$ ) or 
${ }^{3} \mathrm{He}(99.997 \%)$ gas at temperature $T \sim 10 \mathrm{~K}$. We managed to stop antiprotons and form $\bar{p} \mathrm{He}^{+}$within the volume of the chamber at helium density $\rho=3 \times 10^{16} \mathrm{~cm}^{-3}$; this was made possible by minimizing the energy spread and emittance of the antiproton beam through careful tuning of the electron beam cooler of the AD [18], optimizing the trajectory of the antiprotons through the RFQD using microwire beam profile monitors, adjusting the output energy of the RFQD by biasing its electrodes with a high voltage [17], and utilizing a target entrance window of superior thickness uniformity (better than $\pm 100 \mathrm{~nm}$ ) compared to those previously [5] used. The gas pressure (typically $p \sim 3 \times 10^{-2} \mathrm{mb}$ ) was measured using capacitance manometers. Charged pions emerging from antiproton annihilations were detected by Cherenkov counters surrounding the target, equipped with a microchannel plate photomultiplier which measured the envelope of the Cherenkov light with subnanosecond time resolution. By recording the waveform with a digital oscilloscope, delayed annihilation time spectra (the distribution of the number of antiproton annihilations, as a function of time elapsed since the neutral $\bar{p} \mathrm{He}^{+}$was formed) were recorded. A Nd:YAG-pumped dye laser with four dye amplification stages produced 2-4-ns-long laser pulses tuned to wavelengths $\lambda=264-726 \mathrm{~nm}$ with the high energy required here ( $\varepsilon=10-80 \mathrm{~mJ} /$ pulse). In order to irradiate all the antiprotons stopped in the target, the laser beam was expanded to a diameter $d=12 \mathrm{~cm}$ using 15 -cm-diameter lenses and allowed to enter the target through two $12-\mathrm{cm}-$ diameter fused silica windows.

The time spectra of Fig. 2 confirm the reasoning outlined above concerning the tails of spikes in the annihilation distributions. Figure 2(a) was found at the high target density $\rho=2 \times 10^{18} \mathrm{~cm}^{-3}$ by tuning the laser to the wavelength $\lambda=597.3 \mathrm{~nm}$ of a $\bar{p}^{4} \mathrm{He}^{+}$transition from state $(n, \ell)=(39,35)$ with lifetime $\tau_{(39,35)} \sim 1.4 \mu \mathrm{s}$, to a state $(n, \ell)=(38,34)$ with a short Auger lifetime $\tau_{(38,34)}^{A} \sim$ 9 ns $[8,9,16]$. Auger emission (curved arrow in Fig. 1) then produced the $\bar{p} \mathrm{He}^{2+}$ ionic state $\left(n_{i}, \ell_{i}\right)=(32,31)$. The annihilation spike decayed with lifetime $\tau_{\text {obs }} \sim(9 \pm 1) \mathrm{ns}$, which indicates that the ion was destroyed by collisions in a short time relative to the $\bar{p} \mathrm{He}^{+}$Auger lifetime $\tau_{(38,34)}^{A} \sim$ $9 \mathrm{~ns}$. When the same transition was measured at a 100 times lower density $\rho=3 \times 10^{16} \mathrm{~cm}^{-3}$, however, the lifetime increased by an order of magnitude to $\tau_{\mathrm{obs}} \sim$ $(90 \pm 20)$ ns [Fig. 2(b)], due to the prolonged collisional lifetime $\tau_{i}$ of the ion occupying $\left(n_{i}, \ell_{i}\right)=(32,31)$. This spectrum, representing data accumulated from $\sim 10^{10}$ antiprotons stopped in the target, includes $\sim 10^{6}$ such ions. The laser was fired at time $t>2 \mu \mathrm{s}$ after antiproton arrival, to allow the $\bar{p} \mathrm{He}^{+}$to thermalize to the gas temperature $T \sim$ $10 \mathrm{~K}$. The temperature of the ions was inferred from the observed thermal Doppler widths $(\Gamma<500 \mathrm{MHz})$ of the 597.3-nm $\bar{p} \mathrm{He}^{+}$resonance; the $\bar{p} \mathrm{He}^{2+}$ ions produced subsequently have roughly the same temperature, since the kinetic energy of the emitted Auger electron is very low $\left(E_{(38,34)}^{A}=0.8 \mathrm{eV}\right.$, corresponding to the energy difference between the Auger-dominated $\bar{p} \mathrm{He}^{+}$and final $\bar{p} \mathrm{He}^{2+}$ states; see Fig. 1). This results in a $\sim 100-\mu \mathrm{eV}$ recoil energy for the ion.

Further support for the production of ions was provided by populating the same $\bar{p}^{4} \mathrm{He}^{2+}$ state $\left(n_{i}, \ell_{i}\right)=(32,31)$ by driving two other $\bar{p}^{4} \mathrm{He}^{+}$transitions: $(n, \ell)=(40,35) \rightarrow$ $(39,34)$ at wavelength $\lambda=672.8 \mathrm{~nm}$, and $(37,34) \rightarrow$ $(38,33)$ at $713.6 \mathrm{~nm}$. Despite the fact that the three transitions involved intermediate states having very different Auger $[8,9,16]$ lifetimes $(\sim 9 \mathrm{~ns}, \sim 1 \mathrm{~ns}$, and $\sim 3 \mathrm{ps,} \mathrm{re-}$ spectively) and emission energies of the Auger electron $(0.8 \mathrm{eV}, 2.6 \mathrm{eV}$, and $0.5 \mathrm{eV}$, respectively), the observed lifetimes $\tau_{\mathrm{obs}}$ of the tails of the corresponding annihilation spikes agreed with one another at low target densities $\rho<$ $6 \times 10^{17} \mathrm{~cm}^{-3}$, where the ion's lifetime is expected to dominate (i.e., $\tau_{\mathrm{obs}} \sim \tau_{i}$ ). This result is consistent with the expectation that all three $\bar{p}^{4} \mathrm{He}^{+}$transitions ultimately proceed to the same long-lived $\bar{p}^{4} \mathrm{He}^{2+}$ state $\left(n_{i}, \ell_{i}\right)=$ $(32,31)$.

We measured the lifetimes $\tau_{i}$ of $\bar{p}^{4} \mathrm{He}^{2+}$ produced at target densities between $\rho=3 \times 10^{16}$ and $2 \times 10^{18} \mathrm{~cm}^{-3}$ initially occupying four states: $\left(n_{i}, \ell_{i}\right)=(32,31)$ (de-

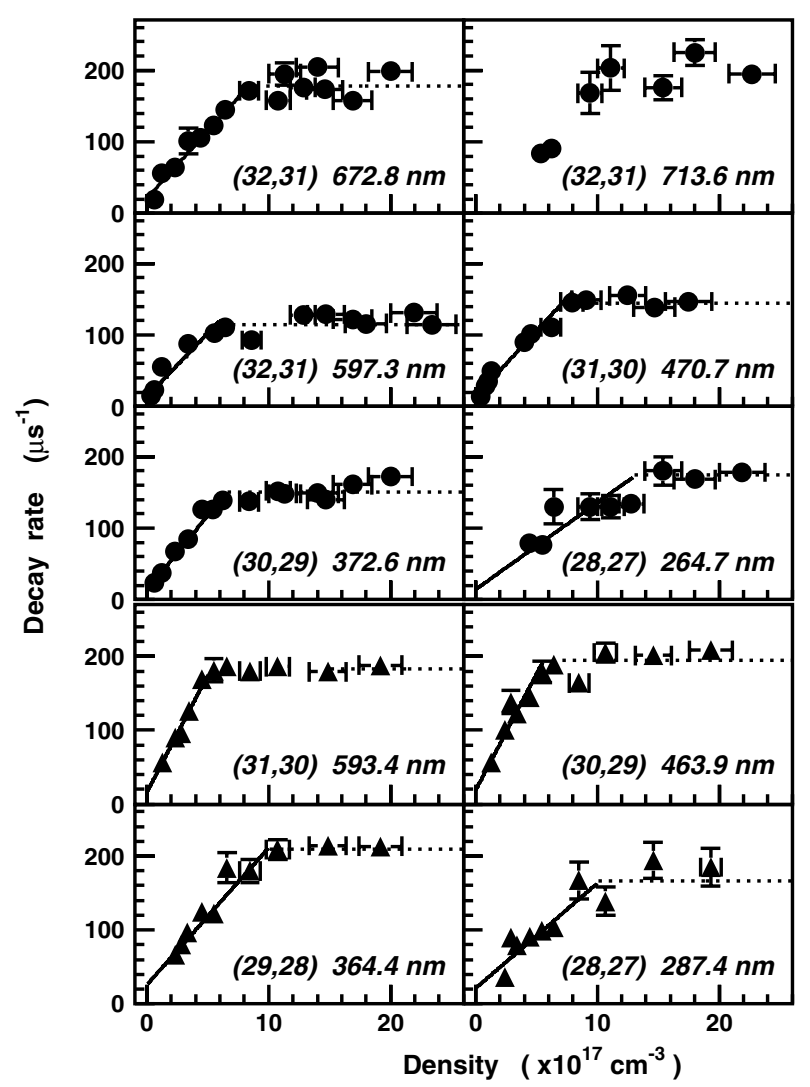

FIG. 3. Decay rates of annihilation signals measured at various target densities. Data corresponding to $\bar{p}^{4} \mathrm{He}^{2+}$ states are indicated by filled circles, and those of $\bar{p}^{3} \mathrm{He}^{2+}$ by triangles. 


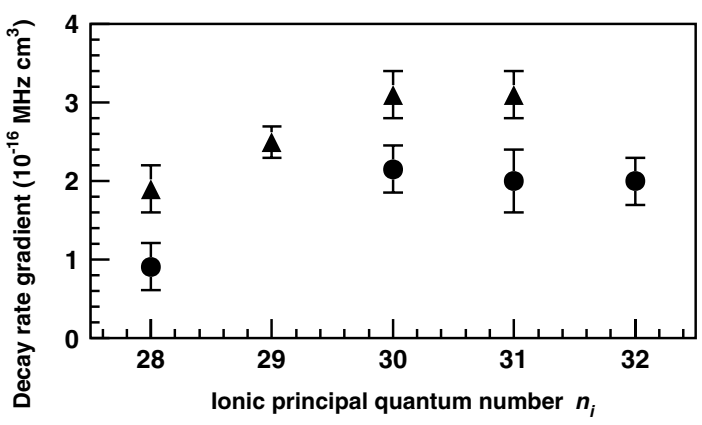

FIG. 4. Gradients of the decay rates per target density of four $\bar{p}^{4} \mathrm{He}^{2+}$ (indicated by filled circles) and $\bar{p}^{3} \mathrm{He}^{2+}$ (triangles) ionic states, as a function of the principal quantum number $n_{i}$.

scribed above), $(31,30)$ (populated by inducing the $\lambda=$ 470.7-nm transition, the intermediate $\bar{p} \mathrm{He}^{+}$state having an Auger lifetime $\sim 5 \mathrm{~ns}),(30,29)(372.6 \mathrm{~nm}$, Auger lifetime $\sim 5 \mathrm{~ns})$, and $(28,27)(264.7 \mathrm{~nm}$, reliable Auger lifetime is unavailable). Four $\bar{p}^{3} \mathrm{He}^{2+}$ states $(31,30),(30,29)$, $(29,28)$, and $(28,27)$ were also measured using the $\bar{p}^{3} \mathrm{He}^{+}$ transitions at $\lambda=593.4,463.9,364.4$, and $287.4 \mathrm{~nm}$, the Auger-dominated states of which had lifetimes of about 3, 1,1 , and 4 ns, respectively. In Fig. 3, the observed decay rates $\gamma_{\mathrm{obs}}$ of the tails of the annihilation spikes are plotted as a function of target density, with the corresponding $\bar{p} \mathrm{He}^{2+}$ ionic state and the wavelength of the $\bar{p} \mathrm{He}^{+}$transition indicated in each case. It is interesting to note that when compared at the same density (e.g., $\rho=$ $4 \times 10^{17} \mathrm{~cm}^{-3}$ ), the collisional decay rates of $\bar{p}^{3} \mathrm{He}^{2+}$ increase for larger $n_{\mathrm{i}}$ values $\left[\gamma_{\mathrm{obs}} \sim 0.09(1), 0.12(3)\right.$, $0.17(1)$, and $0.18(1) \mathrm{ns}^{-1}$ for the ionic states $(28,27)$, $(29,28),(30,29)$, and $(31,30)$, respectively].

In the region $\rho<(5-10) \times 10^{17} \mathrm{~cm}^{-3}, \gamma_{\mathrm{obs}}$ increased roughly as a linear function of density, as indicated by solid lines in Fig. 3. This qualitatively agrees with expectations for $\bar{p} \mathrm{He}^{2+}$ ions destroyed in binary collisions with helium atoms as described by Eq. (3). The weak transition at $\lambda=$ $713.6 \mathrm{~nm}$ could not be observed at densities $\rho<5 \times$ $10^{17} \mathrm{~cm}^{-3}$, and so it is not analyzed here. Although a reliable extrapolation of all these decay rates to $\rho=0$ was not possible due to the large systematic errors associated with measuring gas densities $\rho<3 \times 10^{17} \mathrm{~cm}^{-3}$, the data are consistent with the theoretical expectation of isolated ions having lifetimes of a few hundred nanoseconds against annihilation. At high densities $\rho>1 \times$ $10^{18} \mathrm{~cm}^{-3}$, the $\gamma_{\mathrm{obs}}$ values of four transitions at $\lambda=$ $597.3,470.7,372.6$, and $287.4 \mathrm{~nm}$ saturated to values consistent with the theoretical Auger values $\tau^{A}=4-9 \mathrm{~ns}$ folded with the time resolution $\tau_{\text {las }} \sim 4 \mathrm{~ns}$ of the laser pulse, as indicated by dotted lines in Fig. 3. The other transitions involving shorter Auger lifetimes $\tau^{A}<2 \mathrm{~ns}$ saturated to values corresponding to this resolution $\tau_{\text {las }}$.

The measured gradients $d \gamma_{i} / d \rho$ are plotted as a function of the $n_{i}$ value in Fig. 4. The collisional decay rates of $\bar{p}^{3} \mathrm{He}^{2+}$ were consistently larger than those of $\bar{p}^{4} \mathrm{He}^{2+}$ measured at the same target temperature and pressure; this qualitatively agrees with the expectation of Eq. (3) that $\bar{p}^{3} \mathrm{He}^{2+}+{ }^{3} \mathrm{He}$ collisions have higher quenching rates than $\bar{p}^{4} \mathrm{He}^{2+}+{ }^{4} \mathrm{He}$ due to the smaller reduced mass $M_{\text {red }}$. The $d \gamma_{i} / d \rho$ value in $\bar{p}^{3} \mathrm{He}^{2+}$ increased from $1.9(3) \times 10^{-16}$ to $3.2(3) \times 10^{-16} \mathrm{MHz} \mathrm{cm}^{3}$ for increasing $n_{i}$ from 28 to 31 , whereas the $n_{i}$ dependence appears to be smaller in $\bar{p}^{4} \mathrm{He}^{2+}, d \gamma_{i} / d \rho$ being distributed around $(1-2) \times$ $10^{-16} \mathrm{MHz} \mathrm{cm}^{3}$.

The cross section of ions destroyed by collisions was estimated from the measured decay rates and Eq. (3) as $\sigma \sim(4-10) \times 10^{-15} \mathrm{~cm}^{2}$. This value is an order of magnitude larger than theoretical cross sections $[4,12,13]$ for collisional Stark mixing, but as these theories pertain to $\bar{p} \mathrm{He}^{2+}$ recoiling at temperature $T \sim 10^{4} \mathrm{~K}$, they cannot be directly compared with the present experiment carried out on cold ions. These results indicate that laser spectroscopy of $\bar{p} \mathrm{He}^{2+}$ may be possible, by inducing transitions between ionic states with different $n_{i}$ values and making use of the $n_{i}$ dependence of their lifetimes to detect the resonance signal.

We thank the CERN AB division, cryogenic laboratory, T. S. Jensen, R. Landua, and V. E. Markushin for their help. This work was supported by the Grant-in-Aid for Specially Promoted Research (15002005) of Monbukagakusho of Japan and by the Hungarian Scientific Research Fund (OTKA T046095 and TeT-Jap-4/00).

[1] T. B. Day, Nuovo Cimento 18, 381 (1960).

[2] M. Leon and H. A. Bethe, Phys. Rev. 127, 636 (1962).

[3] H. A. Bethe and E. E. Salpeter, Quantum Mechanics of One- and Two-Electron Atoms (Plenum, New York, 1977).

[4] G. Reifenröther, E. Klempt, and R. Landua, Phys. Lett. B 203, 9 (1988).

[5] M. Hori et al., Phys. Rev. Lett. 91, 123401 (2003).

[6] T. Yamazaki et al., Phys. Rep. 366, 183 (2002).

[7] G. Gabrielse et al., Phys. Rev. Lett. 82, 3198 (1999).

[8] V. I. Korobov, Phys. Rev. A 67, 062501 (2003).

[9] Y. Kino, M. Kamimura, and H. Kudo, Nucl. Instrum. Methods Phys. Res., Sect. B 214, 84 (2004).

[10] W. A. Beck, L. Wilets, and M. A. Alberg, Phys. Rev. A 48, 2779 (1993).

[11] J. S. Cohen, Phys. Rev. A 62, 022512 (2000).

[12] R. Landua and E. Klempt, Phys. Rev. Lett. 48, 1722 (1982).

[13] T. S. Jensen and V. E. Markushin, Eur. Phys. J. D 19, 165 (2002); (private communication).

[14] T. B. Day, G. A. Snow, and J. Sucher, Phys. Rev. Lett. 3, 61 (1959).

[15] E. Borie and M. Leon, Phys. Rev. A 21, 1460 (1980).

[16] H. Yamaguchi et al., Phys. Rev. A 66, 022504 (2002).

[17] A. M. Lombardi, W. Pirkl, and Y. Bylinsky, in Proceedings of the 2001 Particle Accelerator Conference, Chicago, 2001 (IEEE, Piscataway, NJ, 2001), pp. 585-587.

[18] P. Belochitskii, T. Eriksson, and S. Maury, Nucl. Instrum. Methods Phys. Res., Sect. B 214, 176 (2004). 